\title{
A Large Format Surface-Enhanced Raman Spectroscopy Substrate Optimized for Enhancement and Uniformity
}

Katherine N. Kanipe, Philip P. F. Chidester, Galen D. Stucky, Martin Moskovits*

Department of Chemistry \& Biochemistry, University of California Santa Barbara, Santa

Barbara, CA 93106-9510

*email: moskovits@chem.ucsb.edu

This file contains:

- Enhancement factor calculation repeated with 1,2-bis-(4-pyridyl) ethylene (Figure S1)

- A derivation of the quantity $R_{o}=\hat{r}_{o}^{*} \hat{r}_{o}$ as used in the analysis of spectroscopic ellipsometry measurements 


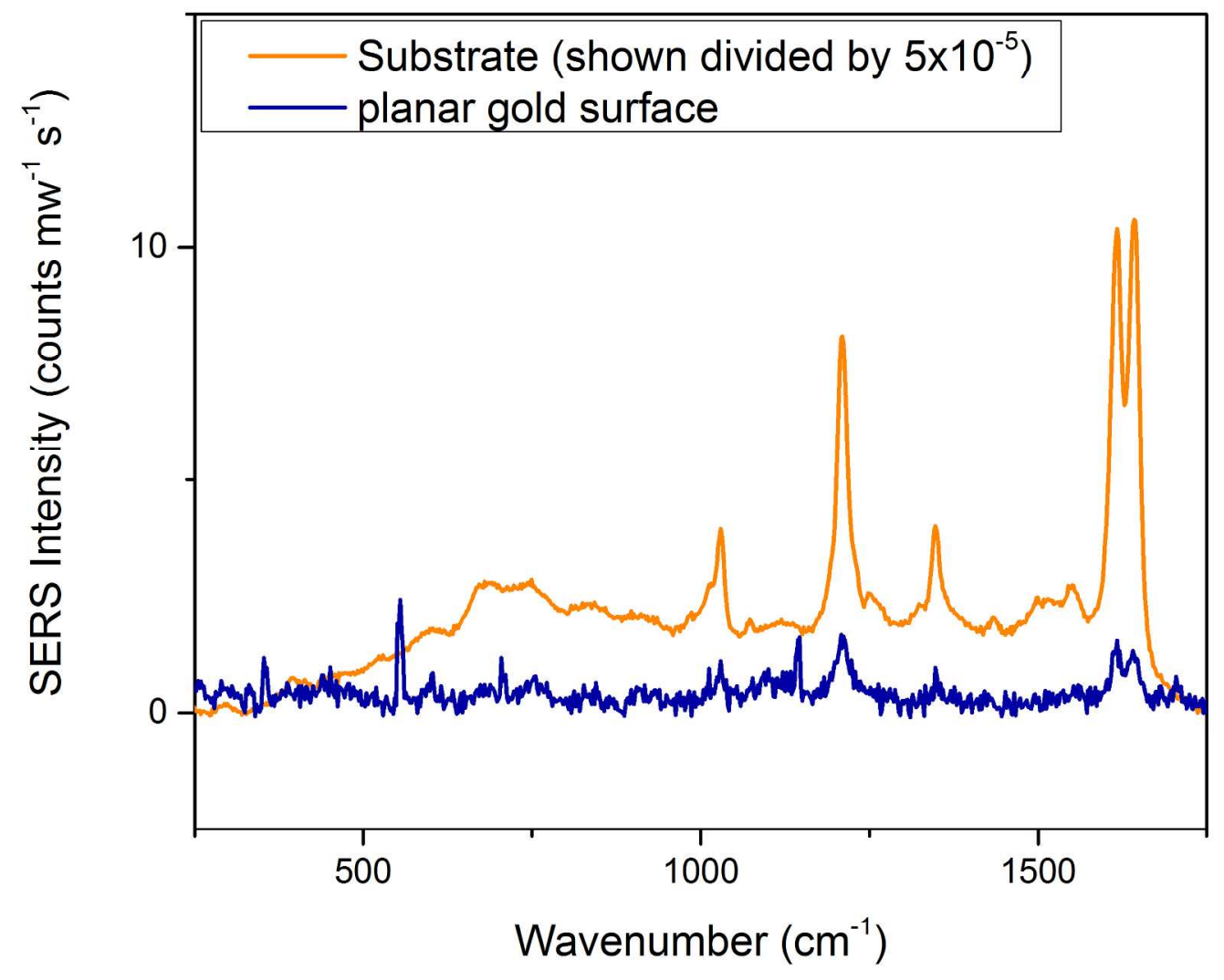

Figure S1. The SERS spectra of 1,2-bis-(4-pyridyl) ethylene adsorbed on a gold SERS substrate and on a planar gold surface. The intensity ratio of the two spectra implies an enhancement factor of $\sim 10^{7}$. The planar gold surface is a $100 \mathrm{~nm}$ thick layer of gold deposited by e-beam evaporation. A few weak, broad features are observed in the spectrum recorded from the planar gold surface in addition to those of BPE. These are likely fluorescence bands of species photosynthesized during the $5 \mathrm{~min}$. exposure time.

\section{A derivation of the quantity $\boldsymbol{R}_{o}=\hat{\boldsymbol{r}}_{o}^{*} \hat{\boldsymbol{r}}_{o}$ as used in the analysis of spectroscopic ellipsometry measurements:}

The gratings produced were considered to be effective optical media since their features are smaller than the wavelength used to excite surface enhanced Raman $(633 \mathrm{~nm})$. Accordingly, one can assume that the media can be described by effective optical constants $(n, k)$. Because the structure of the gratings are somewhat different in the directions normal and tangential to the plane of the grating, in general, one would have to use Fresnel coefficients that relate to a uniaxial anisotropic medium ${ }^{30}$ and also the fact that the grating might be construed to be a "film" on top of a silicon substrate. However, the grating is sufficiently thick so that no light penetrates to the silicon substrate. Accordingly, we assumed the ellipsometric measurements were carried out on a single, infinite medium. We also ignored its anisotropy for two reasons: First we noted 
that the values of $n$ and $k$ returned by analyzing the ellipsometric values at various angles of incident did not vary a great deal, and we used the optical constants returned by the calculation to compute normal incidence reflectance values which depend only on the tangential optical constants, independent of the angle of incidence.

Ellipsometry returns two quantities, often stated as two angles $\Psi$ and $\Delta$ which are related to the complex-valued Fresnel coefficients $\hat{r}_{s, p}$ as follows, $\frac{\hat{r}_{p}}{\hat{r}_{s}}=\rho=\tan \Psi e^{i \Delta}$. (The caret indicates complex values.) The optical constants, $n$ and $k$, of an optically isotropic surface measured at angle of incidence $\theta$, can be calculated from the ellipsometric parameters using the following expression: ${ }^{23}$

$$
(n+i k)^{2}=\sin ^{2} \theta\left[1+\tan ^{2} \theta\left(\frac{1-\rho}{1+\rho}\right)^{2}\right]
$$

The s-polarized reflectance, $R_{s}$, was calculated using the expression $R_{S}=\hat{r}_{S}^{*} \hat{r}_{s}$, in which the asterisk denoted the complex conjugate. And $\hat{r}_{S}$ is the s-polarized Fresnel function:

$\hat{r}_{S}=\frac{\hat{n}_{i} \cos \theta-\hat{n}_{t} \cos \varphi}{\hat{n}_{i} \cos \theta+\hat{n}_{t} \cos \varphi}$

in which $\hat{n}_{t}=n+i k$ as defined above, $\hat{n}_{i}$ is the complex refractive index of the incident medium (approximately equal to 1 , if the medium is air), and $\varphi$ is the complex angle of refraction in the effective medium that can be calculated from the value of $\theta$, using Snell's law. For normal incidence both $\theta$ and $\varphi$ are zero, and the reflectance, $\hat{r}_{o}$ is given by:

$\hat{r}_{o}=\frac{\hat{n}_{i}-\hat{n}_{t}}{\hat{n}_{i}+\hat{n}_{t}}$. The fraction of the incident light energy reflected is $R_{o}=\hat{r}_{o}^{*} \hat{r}_{o}$, which will be a function of the wavelength. Therefore the fraction of the incident energy absorbed will be $A_{o}(\lambda)=1-R_{o}(\lambda)$. Spectra of $A_{o}(\lambda)$ are shown in Fig. $3 \mathrm{~b}$ of the main text. 